\title{
Studies of Relativistic Jets in Active Galactic Nuclei with SKA
}

\author{
Iván Agudo*1 ${ }^{* 1}$ Markus Böttcher ${ }^{2}$, Heino Falcke ${ }^{3}$, Markos Georganopoulos ${ }^{4}$, Gabriele \\ Ghisellini $^{5}$, Gabriele Giovannini ${ }^{6}$, Marcello Giroletti ${ }^{7}$, Jose L. Gómez ${ }^{8}$, Leonid \\ Gurvits $^{1,9}$, Robert Laing ${ }^{10}$, Matthew Lister ${ }^{11}$, Jose-María Martíi ${ }^{12}$, Eileen T. Meyer ${ }^{13}$, \\ Yosuke Mizuno ${ }^{14}$, Shane O'Sullivan ${ }^{15}$, Paolo Padovani ${ }^{10}$, Zsolt Paragi ${ }^{1}$, Manel \\ Perucho $^{12}$, Dominik Schleicher ${ }^{16}$, Łukasz Stawarz ${ }^{17}$, Nektarios Vlahakis ${ }^{18}$, and John \\ Wardle $^{19}$
}

${ }^{1}$ Joint Institute for VLBI in Europe; ${ }^{2}$ North-West University; ${ }^{3}$ Radboud University Nijmegen;

${ }^{4}$ University of Maryland; ${ }^{5}$ INAF - Osservatorio Astronomico di Brera; ${ }^{6}$ Università di Bologna;

${ }^{7}$ INAF - Istituto di Radioastronomia; ${ }^{8}$ Instituto de Astrofísica de Andalucía - CSIC; ${ }^{9}$ Delf University of Technology; ${ }^{10}$ European Southern Observatory; ${ }^{11}$ Purdue University;

${ }^{12}$ Universitat de València; ${ }^{13}$ Space Telescope Science Institute; ${ }^{14}$ National Tsing-Hua University; ${ }^{15}$ The University of Sydney; ${ }^{16}$ Georg-August-Universität Göttingen; ${ }^{17}$ Institute of Space and Astronautical Science-JAXA; ${ }^{18}$ University of Athens; ${ }^{19}$ Brandeis University E-mail: agudo@jive.nl

Relativistic jets in active galactic nuclei (AGN) are among the most powerful astrophysical objects discovered to date. Indeed, jetted AGN studies have been considered a prominent science case for SKA, and were included in several different chapters of the previous SKA Science Book (Carilli \& Rawlings 2004). Most of the fundamental questions about the physics of relativistic jets still remain unanswered, and await high-sensitivity radio instruments such as SKA to solve them. These questions will be addressed specially through analysis of the massive data sets arising from the deep, all-sky surveys (both total and polarimetric flux) from SKA1. Wide-field verylong-baseline-interferometric survey observations involving SKA1 will serve as a unique tool for distinguishing between extragalactic relativistic jets and star forming galaxies via brightness temperature measurements. Subsequent SKA1 studies of relativistic jets at different resolutions will allow for unprecedented cosmological studies of AGN jets up to the epoch of re-ionization, enabling detailed characterization of the jet composition, magnetic field, particle populations, and plasma properties on all scales. SKA will enable us to study the dependence of jet power and star formation on other properties of the AGN system. SKA1 will enable such studies for large samples of jets, while VLBI observations involving SKA1 will provide the sensitivity for pc-scale imaging, and SKA2 (with its extraordinary sensitivity and dynamic range) will allow us for the first time to resolve and model the weakest radio structures in the most powerful radio-loud AGN.

Advancing Astrophysics with the Square Kilometre Array

June 8-13, 2014

Giardini Naxos, Italy

*Speaker.

${ }^{\dagger}$ On behalf of the SKA Cosmic Magnetism Working Group 


\section{Introduction}

Relativistic jets in AGN are among the most powerful astrophysical objects so far discovered. Their relativistic nature causes them to emit abundant and extremely time-variable radiation in all spectral ranges from radio wavelengths to gamma-rays, whereas bulk relativistic motion leads to strong Doppler boosting effects, making them detectable at extreme cosmological distances. In AGN, relativistic plasma jets are thought to be formed as the result of accretion onto super-massive black-holes $(\mathrm{SMBH})$ in the presence of rotating accretion disks and co-rotating magnetic fields. The study of the details involved in the jet formation process, which are still largely unknown, makes AGN jets a powerful tool to probe the environment of objects in extremely compact matterstates, and the physics of high-energy plasmas and their magnetic fields.

During recent years, a drastic improvement in our understanding of relativistic jets has been enabled by advances in supercomputing facilities for state-of-the-art numerical simulations, as well as deep, high-resolution radio, optical-IR and X-ray imaging and the advent of comprehensive monitoring programs covering most accessible spectral ranges from radio to gamma-rays. However, most of the fundamental questions about the physics of relativistic jets still remain unanswered, and await high sensitivity radio instruments such as SKA to solve them. These questions include: a) why are jets produced efficiently only in some systems, and what is the relation between jet power and the properties of the black hole and accretion system? b) what influence do magnetic fields have on jet formation, collimation, and maintenance up to distances $>100 \mathrm{kpc}$ ? c) what is the actual plasma composition at different jet scales and how does it evolve down the jet? d) how does the particle acceleration mechanism make the jet an efficient emitter on scales exceeding the size of the host galaxy? and d) how does the feedback between the jet and the (inter-)galactic medium influence the evolution of galaxies and clusters, and how do AGN jets and their central black holes evolve on cosmological time scales up to $z \sim 10$ ?

These questions will perhaps be addressed first by analysis of the massive data sets arising from deep all-sky surveys with SKA1, including both total and polarimetric flux. In particular, SKA1 studies of relativistic jets at different resolutions will allow, for the first time: i) studies (including imaging) of AGN jets throughout cosmic time, back to the epoch of re-ionization; ii) robust jet composition studies through unparalleled circular polarization (CP) sensitivity/purity; iii) an understanding of the origin of intrinsic differences in jet power in AGN via imaging of thousands of radio-weak AGN; iv) a characterization of the three-dimensional distributions of flow parameters such as velocity, proper emissivity, and magnetic field structure (through total flux and polarization imaging), that lead to estimates of key physical quantities such as mass and energy fluxes and entrainment rates; v) a probe of the magnetoionic environments of the jets (perhaps including their confining fields) through deep full Stokes (and Faraday rotation measure) imaging at all scales along and across a large number of objects; and vi) an understanding of the interplay between the jet and the ambient medium, both in the local universe and along cosmic time up to $z \sim 10$.

In $\S 2-10$, we describe the current status of the main research areas involving jetted AGN, and the requirements necessary for SKA1 to advance in these fields. In $§ 11$ and 12 we summarize the general need for future jetted AGN studies with SKA1, and we discuss the prospects for the SKA1 early science phase, as well as for SKA2 on AGN jet investigations. 


\section{Jet Formation, Collimation, and Evolution}

Models proposed to explain the origin of relativistic jets in AGN involve accretion, in the form of a disk, of interstellar mass and gas from tidally disrupted stars onto a supermassive black hole. There is a general agreement that magnetic fields play a crucial role in this process. In the models of magnetically driven outflows, poloidal magnetic fields anchored at the base of the accretion disk generate a toroidal component and a subsequent poloidal electromagnetic flux of energy (Poynting flux) that accelerates the magnetospheric plasma along the poloidal magnetic field lines (see Blandford \& Payne 1982; Li et al. 1992, and Fig. 1 for illustration). Energy can also be extracted from rotating black holes with similar efficiencies by the Blandford \& Znajek (1977) mechanism. The strength of the magnetic field on the event horizon can be estimated to be of the order of thousands of Gauss. How this magnetic field is built up from the disk magnetic field is the subject of current research (Tchekhovskoy et al. 2011; McKinney et al. 2012).

Since in any version of a magnetic central engine the accretion disk is magnetized, we expect a wind to be driven from its surface (close to the black hole) with a power comparable to the Blandford-Znajek power. If the mass loading of disk winds (mainly formed by electron-proton plasma) were too high to produce ultrarelativistic terminal speeds, both mechanisms would need to operate at the same time, producing stratified jets in both composition (outer electron-proton wind, inner electron-positron jet) and speed. On the other hand, the Blandford-Znajek mechanism does not provide any collimation. Hence the central engine must provide an additional ingredient to confine the outflow until it reaches the superfast magnetosonic regime. Multi-wavelength fullpolarization (4-Stokes) studies with SKA1 at all resolutions, down to the milli-arcsecond (mas) scale with SKA1-VLBI (Paragi et al. 2015), including CP observations with precision up to $0.01 \%$, are ideal to test the above mentioned jet/disc-wind scenario, and therefore of the role of such winds in the collimation of jets (see $\S 4$ ). Furthermore, full-polarization measurements would allow us to determine the fraction of electron-positron pairs, the shape of the low energy distribution of the relativistic emitting particles and the presence of a dynamically dominant proton component, helping to constrain the initial magnetization of the wind, a very important parameter in the magnetic theory of AGN jets determining the asymptotic speed of the flow.

Theoretical and observational considerations suggest that jets must remain Poynting dominated along the acceleration phase. The success of magnetic acceleration (Vlahakis \& Königl 2004) has been confirmed in time-dependent numerical simulations (Komissarov et al. 2007). In the case of parabolic jets, the acceleration continues until the jet becomes particle-dominated, at sub-parsec scales (hundreds to thousands of black hole gravitational radii). To stablish the exact location of the site of this conversion and its possible observational signature, in particular its position in relation to the radio core, is a subject of current active research for multi-spectral-range observational campaigns where high-frequency polarimetric single-dish and VLBI observations are key ingredients. With its superb sensitivity, SKA1-MID in Band 5 (both as a VLBI station or not) will allow these kinds of studies for a large number of sources seen from the southern hemisphere, where a growing number of panchromatic facilities are expected to operate with SKA. These studies are particularly interesting for nearby sources such as Centaurus A and M 87, where the highest linear resolutions will be achieved to resolve the jets both across and along their axis, from the regions close to the SMBH to those where the Poynting flux converts to particle dominated plasma. 


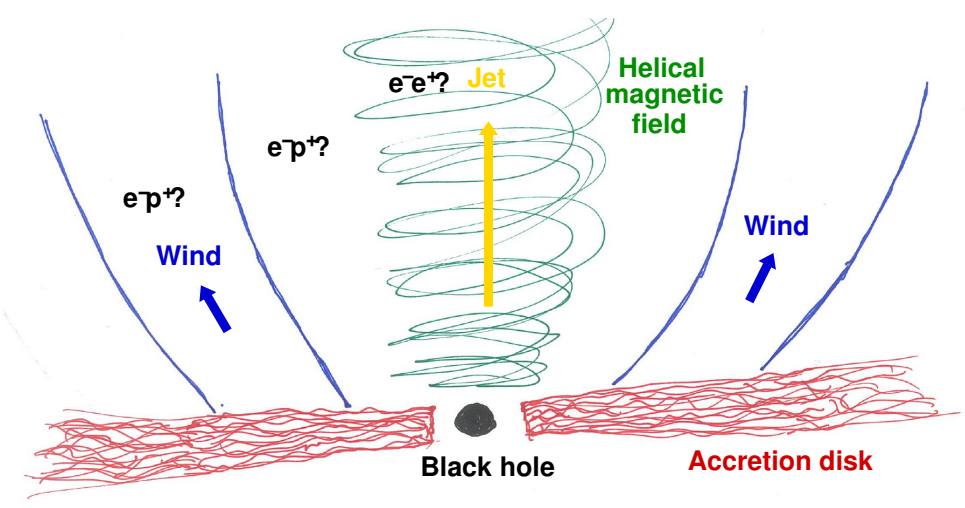

Figure 1: Conceptual illustration of the jet formation and collimation scenario described in $\S 2$.

\section{Particle Acceleration in Jets}

The ambiguity of the location where Poynting-flux dominated outflows are converted into kinetic dominated jets reflects a general lack of understanding of the particle acceleration processes which are responsible for maintaining power-law distributions of radiating ultra-relativistic electrons over decades of length scales along the jet. These scales range from the sub-pc to pc scale, where the high-energy emission from blazars is likely produced, to the kpc scale where the jets terminate. In the case of low plasma magnetization, diffusive acceleration taking place at the fronts of strong hydrodynamical shocks (see e.g., Summerlin \& Baring 2012, and references therein) could be considered as the most efficient way of energizing jet particles, leading naturally to the formation of power-law spectra. In the case of a high magnetization, on the other hand, relativistic magnetic reconnection (e.g. Sironi \& Spitkovsky 2014) and stochastic interactions of particles with magnetic turbulence (Stawarz \& Petrosian 2008, and references therein) are expected to play a major role. Although the relativistic nature of AGN jets constitutes a difficulty in this context (the aforementioned particle acceleration processes are well understood only in a non-relativistic regime), the slope and normalization of the resulting non-thermal particle spectra depend strongly on the underlying physical conditions in all of these three cases. This offers the opportunity to exploit high-quality observational data to determine the underlying non-thermal particle distributions, which can be used to constrain the acceleration processes at work.

Good-quality radio data provided by SKA1 has the potential to reveal the spectral shapes of freshly accelerated jet electrons. In the case of the extended arcsecond-scale jets and jet cocoons (lobes), such studies are expected to be free of any absorption effects even at low frequencies $(<1-2 \mathrm{GHz})$. The superior SKA1 sensitivity in full polarimetric mode (at $\gtrsim 1 \mathrm{GHz}$ ), combined with simultaneous SKA1-VLBI observations, will enable spatially resolved spectral studies of the radio emission of the innermost regions of AGN jets out to large redshifts, $(z \sim 5-10)$. Additional constraints on the acceleration sites of the highest-energy electrons and their radiation environment, based on co-ordinated multi-wavelength observations at high radio frequencies (see previous section), will be facilitated by the location of the SKA1-MID in South Africa, in close vicinity to world-class optical (SALT) and very-high-energy $\gamma$-ray (H.E.S.S. and possibly CTA-South) observatories, in combination with satellite-based X-ray and $\gamma$-ray (Fermi) observations. 


\section{Jet Composition through Circular Polarization Studies}

Circular polarization observations (Stokes $V$ ) of relativistic jets enable us to measure quantities that are not accessible by observation of the first three Stokes parameters $(I, Q, U$, representing total intensity and linear polarization, LP). These include direct measurement of the magnetic field strength, as well as the vector-ordered part of the magnetic field, and hence the true magnetic flux carried by the jet (a conserved quantity that should be equal to the magnetic flux at the central engine). CP will also enable measurements of the composition of the jet (i.e. the $\mathrm{e}^{+}-\mathrm{p}^{+}$ratio) and the low-energy cut-off of the relativistic electron energy distribution (see Homan et al. 2009, where all these physical quantities were estimated for the quasar 3 C 279). These quantities are fundamental to understanding the physical properties of relativistic jets and in particular how they are launched (see $\S 1$ and 2, and Fig. 1), and also for calculating the energy carried by the jet and hence for studying AGN feedback. These have long been open questions in AGN physics.

CP is difficult to study in AGN because it is typically weak ( $\sim 0.1-1 \%$ of Stokes $I)$ and highly variable. The high sensitivity and large instantaneous bandwidths of the SKA1 make it the ideal instrument for measuring CP (and its frequency dependence up to Band 5 of SKA1-MID), as long as the SKA retains excellent polarization purity at all frequencies. As very few circularly polarized AGN are currently known $(<100)$, a large-area CP-sensitive SKA1 survey across all SKA1 bands from 1 to $20 \mathrm{GHz}$ will revolutionize this field of study (see O'Sullivan et al. 2013, for an example of what can be achieved with broadband $\mathrm{CP}$ observations). After finding the CP-brighter sources, SKA1-VLBI is required for follow-up observations to resolve the CP emission region and extract the key jet physical parameters (power, composition, magnetic flux).

$\mathrm{CP}$ measurements are unique in their ability to provide a direct measurement of the jet magnetic field strength, if the field vector ordering can be determined. One situation where there must be a vector-ordered field is if the jet carries an electric current, as in several of the electromagnetic mechanisms for launching jets. Indeed, when combined with LP and Faraday rotation measurements, several fundamental properties of the jet can be measured using CP. For example, when a transverse CP gradient and an RM gradient are both detected, they should have the same sign, each confirming the other, allowing direct measurement of the strength of the toroidal component of the magnetic field and hence the magnitude of the jet current, as well as the low energy cutoff of the electron energy distribution (from the RM-CP ratio). Since we are trying to detect very small fractional polarizations, our detected sources will be bright in Stokes $I$. Thus, as long as SKA1 receivers have excellent polarization purity ( $\sim 0.1 \%$ and $\sim 0.01 \%$ for LP and CP, respectively), then even a shallow large-area early-science survey will dramatically improve our knowledge of $\mathrm{CP}$ in AGN jets. Sources detected in CP could then be followed up with SKA-VLBI to produce groundbreaking advances in our knowledge of magnetic fields and particle populations in jets.

\section{From the Most Powerful to the Weakest Jets}

\subsection{The Radio-loud/Radio-quiet AGN Issue}

AGN come mainly in two classes: radio-loud (RL) AGN, emitting most of their energy over the entire electromagnetic spectrum non-thermally through powerful relativistic jets, and radioquiet (RQ) AGN, whose multi-wavelength emission is dominated by thermal emission, directly 


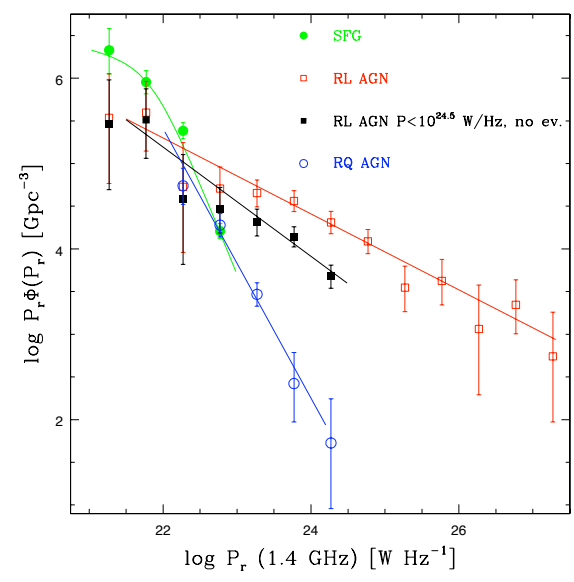

Figure 2: Local $1.4 \mathrm{GHz}$ differential luminosity functions for star forming galaxies and AGNs measured from the Chandra Deep Field South VLA survey. Reproduced from Padovani et al. (2011).

or indirectly related to the accretion disk ${ }^{1}$. The mechanism responsible for radio emission in RQ AGN has been a matter of debate for the past fifty years. Alternatives have included a scaled down version of the RL AGN mechanism (e.g., Ulvestad et al. 2005), star formation (Sopp \& Alexander 1991), and many others. Very recent results on the evolution and luminosity function of very faint radio sources, based on the Chandra Deep Field South samples including a few hundred sources down to $\sim 6-10 \mu \mathrm{Jy} \mathrm{rms}$, suggest close ties between star formation and radio emission in RQ AGN, at least at $z \sim 1.5-2$ (Padovani et al. 2011, Fig. 2, and Padovani et al. in prep.). This is further confirmed by the comparison of the star formation rates derived from the far-IR and radio luminosities, assuming that all the radio emission is due to star formation. For RQ AGN and star forming galaxies the two star formation rate estimates are fully consistent, while for RL AGN the agreement is poor due to the large contribution of the relativistic jet to their radio luminosity (Bonzini et al., submitted). SKA will contribute in two different ways to examine this problem, i) by using the data from the full-polarimetric commensal wide and deep surveys that are being planned for SKA1 in Band 3, which will provide larger samples of RQ and RL AGN covering a wide range of redshifts and powers, and ii) by carrying out polarimetric, wide-field SKA1-VLBI survey follow-up observations in Bands 3 and 5 of the brighter sources ( $\gtrsim 50 \mu \mathrm{Jy}$ ) to distinguish between jet and star formation emission (see $\S 10$ ).

\subsection{Jet Power Along the Radio-loud Classification}

The more powerful radio-loud AGN jets are traditionally divided into Fanaroff-Riley classes FRI and FRII, with the latter having highly collimated jets that terminate in a bright hotspot, indicative of high-Mach number, relativistic flows (Fanaroff \& Riley 1974). Since the pioneering work of Rawlings \& Saunders (1991) on radio-loud AGN, that showed that the jet power correlated with the narrow line luminosity, we have reached the firm conclusion that the jet power correlates with the accretion rate. The present large sample of spectroscopically observed radio-loud objects (SDSS), and blazars detected in the $\gamma$-ray band reinforced this conclusion (Sbarrato et al. 2012a), and led to

\footnotetext{
${ }^{1}$ See Bonzini et al. (2013) for a recent definition of radio-quiet and radio-loud AGN
} 


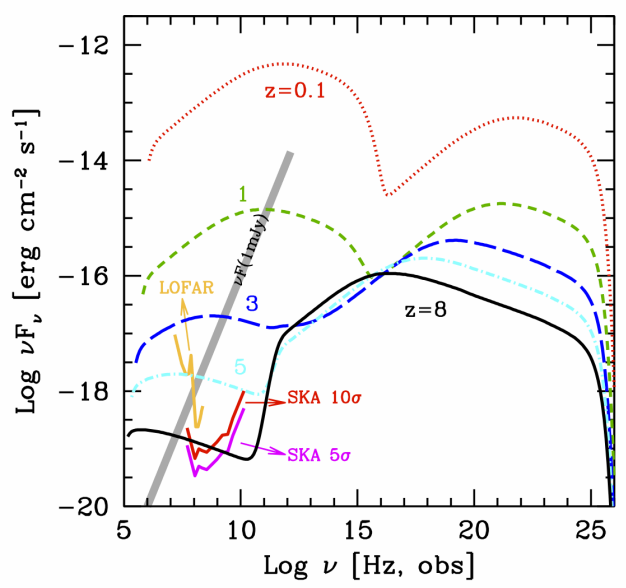

Figure 3: Spectral energy distribution of a typical radio loud quasar at different redshifts as seen in the observer's frame. As redshift increases, the radio spectrum gets progressively more dimmed both because of the larger distance to the source and because of inverse Compton cooling of the particle population responsible for the radio emission. The red and pink continuous lines mark the $10 \sigma$ and $5 \sigma$ limits of SKA1 (LOW and MID), respectively, for $30 \mathrm{~min}$ integration time, whereas the thick grey line correspons to $F(v)=1 \mathrm{mJy}$. Reproduced from Ghisellini et al. (2014).

the idea that low power radio-loud AGN are associated with radiatively inefficient disks, emitting a small amount of ionizing photons, thus explaining why the broad emission lines are so intrinsically weak in these objects (Sbarrato et al. 2012b). Of course, black hole mass and black hole spin are also thought to play key roles (e.g., Blandford \& Znajek 1977; McNamara et al. 2011) by controlling the accretion rate, whereas the external environment also has a strong influence on jet luminosity, as witnessed by 'hybrid' AGN, which exhibit FR I and FR II morphology jets on either side of the active nucleus (Gopal-Krishna \& Wiita 2000).

The weaker flavor of radio-loud AGN have a synchrotron emission peaking in the far-UV and X-ray bands, and are radio-weak (but not radio-quiet). To study them at moderate redshifts $(z>0.4)$, it is crucial to measure the IR and optical cosmic background and to constrain the cosmic magnetic field. The large and deep commensal surveys planned for SKA1 will be an extremely useful tool to help studying weak radio AGN associated with TeV emitters observed with CTA (see Giroletti et al. 2015), and to provide a better characterization of the large scale properties of the cosmic magnetic field (Johnston-Hollitt et al. 2015). Moreover, a significant fraction of Seyfert galaxies have radio structures on scales of $\sim 1 \mathrm{kpc}$ consistent with weak jets being decelerated by the host galaxy's ISM (Gallimore et al. 2006). High-sensitivity, high-resolution full polarimetric studies with SKA1 and SKA1-VLBI have the potential for imaging many of these sources, to shed new light on the long standing problem of radio-loud/radio-quiet AGN through high resolution imaging of nearby sources.

On the opposite power end, powerful jets are associated with high rates of accretion (Ghisellini et al. 2010). The most powerful objects are therefore those jets associated with high accretion and large SMBH masses. Exploiting the relativistically enhanced jet emission, high power blazars can 
be an important tool to explore the far Universe, to find the youngest and most active SMBH, that can constrain (and possibly challenge) current ideas of black hole formation, growth, and feedback (see e.g., Volonteri et al. 2011). For each detected blazar, there must be hundreds of similar sources whose jet is pointing elsewhere. It is this that makes the search for high-redshift blazars with large SMBH masses rewarding. To this task, deep SKA1 surveys at Band 3 or lower frequencies will be crucial. Not only because fluxes weakens with distance, but because, at high $z$, the cosmic background radiation $\left(\mathrm{CMB}\right.$, whose energy density $\left.\propto(1+z)^{4}\right)$, makes the relativistic electrons emit more through the inverse Compton scattering than through the synchrotron process. As a result, the fluxes of powerful blazar jets could be very weak at radio wavelengths (see Ghisellini et al. 2014, and Fig. 3), but still detectable for the SKA1 below $3 \mathrm{GHz}$ for reasonably short integrations ( $30 \mathrm{~min}$ ) up to redshifts $z \sim 8$ and perhaps larger. Deep follow-up Band 3 SKA1-VLBI observations of the first AGN detected will enable studies of the jet morphologies at very high redshifts, and therefore their dense environments. This is also required to discover how the CMB modifies the appearance of radio lobes at high- $z$. SKA1 can therefore revise the fraction of radio-loud to radioquiet AGNs at all redshifts, with a strong impact not only on the radio-loud luminosity function and evolution, but, in general, on the feedback on the host galaxy (through the so called radio-mode).

\section{Detailed Estimates of Fundamental Jet Parameters}

Deep, multifrequency, transverse-resolved radio observations are crucial to the estimation of flow variables in jets. This section concentrates on two topics that are fundamental to jet formation and propagation: velocity fields and magnetic-field topology. One powerful observational technique exploits the intrinsic symmetry of relativistic jet formation. The observed differences between approaching and receding jets are then dominated by the effects of relativistic aberration (Aloy et al. 2000), so by fitting parametrized models to images of both jets in Stokes $I, Q$ and $U$, it is possible to derive the jet inclination and the three-dimensional variations of velocity, rest-frame emissivity and magnetic-field ordering (Laing \& Bridle 2014). A second technique, which can use the same observations, is to analyse the wavelength dependence of linear polarization (and hence internal and external Faraday rotation) across the observing band using RM synthesis. These methods can be used on any scale, given adequate sensitivity and angular resolution. Structural changes on pc scales on timescales of days-years can be monitored, allowing direct measurement of proper motions and the variability of polarization and Faraday rotation structures.

\subsection{Velocity Field}

Low-luminosity (FR I) jets have been shown to decelerate on kpc scales, developing transverse velocity gradients indicative of boundary-layer entrainment (see Laing \& Bridle 2014; Laing 2015). Further progress requires modeling of large samples, to average over intrinsic asymmetries, to explore the dependence of deceleration on jet power and environment and to assess the relative importance of mass injection by stars and boundary-layer entrainment. Resolutions in the range $0.1-0.5$ arcsec and rms noise levels of $0.1-0.5 \mu \mathrm{Jy} /$ beam at $\mathrm{GHz}$ frequencies are needed to observe a sufficiently large number of sources $(\sim 300)$ with SKA1-MID, Bands $3-5$.

The velocity fields of the narrower kpc-scale jets in powerful sources are much less well determined. Here, a key question is whether they have the very fast $(\Gamma \approx 5-10)$ spines needed to gen- 
erate X-ray emission by inverse Compton scattering of CMB photons as well as slower boundary layers. To answer this question, much better resolution and sensitivity are needed $(\approx 0.01 \mathrm{arcsec}$, $\approx 10 \mathrm{nJy} / \mathrm{beam}$ and dynamic range $>10^{7}: 1$; SKA2 Band 4 and 5). See Laing (2015) for a more detailed use case.

The increased sensitivity of SKA1-VLBI would in principle allow the technique to be extended to pc scales. Stratification in the total and linearly polarized emission across the jet width are expected in case the synchrotron emitting jet is threaded by a helical magnetic field (Laing 1981; Aloy et al. 2000). In this case, variations in the velocity field can be estimated by searching for changes in the emission stratification along the jet. Such variations have been observed in the jet of 3C 273 - for which there is evidence for the existence of a helical magnetic field (Asada et al. 2008) - by combining data from 48 epochs spanning 14 years of VLBA observations at $15 \mathrm{GHz}$ (see Gómez et al. 2012, and Fig. 4). A similar jet behavior was already observed with $5 \mathrm{GHz}$ VLBI by Lobanov et al. (2001). A change in the side of the jet that is brighter suggests an acceleration of the emitting plasma, in agreement with the observed acceleration of the pattern velocity associated with superluminal components (Jorstad et al. 2005; Lister et al. 2009). The unprecedented sensitivity of SKA1-VLBI will allow us to extend these studies to statistically significant samples of AGN jets.

For sources in which both jets are visible it is also possible to estimate the flow speed on scales where the pattern speeds of moving components can also be measured. If pattern speeds can be measured for components emitted simultaneously in the approaching and receding jets (as can be done for some microquasar jets), then additional constraints can be derived. M 87 is a particularly important target, as the hypothesis of gradual MHD acceleration on scales $\gg R_{\text {Schwarzschild }}$ can be tested (see e.g. Asada et al. 2014) . Note, however, that the maximum baseline and observing frequency both need to be high enough to ensure transverse resolution of the jets and removal of any associated Faraday rotation (Kovalev et al. 2007), which makes polarimetric VLBI involving SKA1-MID up to Band 5 the ideal option.

If the velocity field, geometry and external environment of a jet are well characterized, then it is possible to apply conservation laws to derive the fluxes of energy, mass and momentum and the variation of entrainment rate with distance from the AGN (Laing \& Bridle 2002).

\subsection{Magnetic Field Topology}

Most models of jet formation require a vector-ordered (toroidal or helical) field, but this has proved to be difficult to confirm observationally. The alternative of a random field made anisotropic by compression (e.g. shocks) or shear often provides at least as good a fit to observations of linear polarization. There are two key diagnostics. Firstly, a helical field with a significant poloidal component shows characteristic asymmetries in its transverse intensity and polarization emission profiles (Aloy et al. 2000). Secondly, if thermal matter is present within the jet volume, then systematic transverse Faraday rotation gradients are expected (Laing 1981; Broderick \& McKinney 2010). Both diagnostics are complicated by effects such as jet bending and foreground Faraday rotation. The best-observed cases on pc scales, 3C 120 (Gómez et al. 2011) and 3C 273 (Asada et al. 2008) suggest a slow foreground screen and jet-related Faraday rotation, respectively. Deep and transverse-resolved observations of the pc-scale emission from a large sample of straight jets are needed, to answer the following questions: Are Faraday rotation gradients preferentially orthogonal to the jet? Does RM synthesis evidence the mixing of thermal and emitting plasma? What are the 


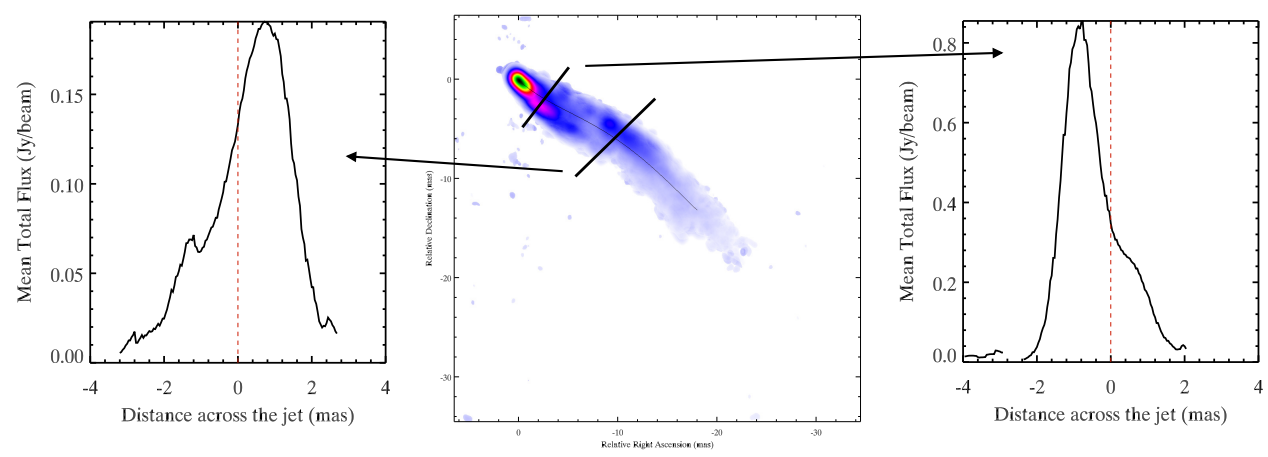

Figure 4: Mean total intensity map of 3C 273 obtained by combining 48 epochs spanning 14 years of VLBA observations at $15 \mathrm{GHz}$ (including data from the MOJAVE program Lister et al. 2013). Left and right panels show slices at 3.1 and 14 mas from the core. A black line extending from the core indicates the jet ridge-line.

relative senses of gradients in the approaching and receding jets? Are transverse emission profiles consistent with the observed rotation? These questions require SKA1-VLBI on Band 5.

The dominant field component on kpc scales in FR I jets is toroidal, but it is unclear whether it is a vector-ordered remnant of the original confining field. If so, systematic and symmetrical gradients in Faraday rotation across the jets are expected. Isolating these from confusing effects of Faraday rotation by the IGM should be feasible for large samples of jets in sparse environments.

\section{Understanding Jet Emission at Sub-pc and Multi-kpc Scales}

Relativistic jets in AGN carry enormous amounts of kinetic energy out from the central black hole into the kpc-scale galaxy and intercluster medium. Most of the energy dissipation in powerful blazars is through the emission of $\mathrm{GeV}$ photons via inverse Compton scattering. However, we lack a clear understanding of where this dissipation takes place. Is it very near to the black hole engine (sub-pc scale) or much further out, beyond the regions dominated by broad-line emitting clouds and the obscuring torus of the unified model? As these scales can differ by orders of magnitude, the answer gives important clues for constraining jet formation and collimation models.

The dominant view during the EGRET era in the 1990's was that the gamma-ray emission is taking place within the sub-pc scale broad line region (Sikora et al. 1994). However, recent multi-wavelength monitoring of a few bright sources from radio to the Fermi GeV band, strongly suggests that in at least some cases, the emission takes place at distances of several $p c$ from the central engine. The key evidence for this is VLBI observations showing that (some) major $\gamma$-ray flares take place at the same time with the ejection of superluminal components from the radio core, located several pc downstream of the central engine (e.g. Marscher et al. 2010), or even co-spatial with VLBI components downstream of the core (e.g. Agudo et al. 2011).

The SKA1-MID observational setup will have suitable features to monitor a substantial number of jets with the ideal sensitivity in total intensity and polarization, angular resolution, and time cadence: in particular Band 5 receivers in VLBI mode (Paragi et al. 2015) will provide $\sim$ milliarcsecond resolution, which corresponds to $\sim$ parsec scales for a broad redshift range. The polarization capabilities will be particularly relevant: current work suggest that polarized features can help 
in the identification and tracking of jet components and in the characterization of the magnetic field evolution in relativistic jets. Only a massive project based on SKA data, perhaps employing similar observing methods and external multi-spectral range infrastructures as other transient objects (see Fender et al. 2015), will allow us to put these indications on firm grounds.

On much larger (kpc) scales, Chandra showed that knots in the jets of powerful quasars are strong X-ray emitters, with the X-ray emission being a separate spectral component from the radioIR-optical one (first seen in PKS 0637-752, Schwartz et al. 2000; Chartas et al. 2000). The standard interpretation is that the $\mathrm{X}$-rays are from inverse Compton scattering, assuming that the radio emitting electron distribution extends down to electron Lorentz factors $\gamma \sim 10-100$ and that the jets are highly relativistic at kpc scales (bulk Lorentz factors $\Gamma \sim 10-20$, Tavecchio et al. 2000; Celotti et al. 2001). This mechanism requires a jet power comparable to or higher than the Eddington luminosity and jets that are very well aligned to the line of sight and have lengths sometimes exceeding $1 \mathrm{Mpc}$. An alternative interpretation is that the X-ray emission is synchrotron coming from a second population of electrons (for a review see Harris \& Krawczynski 2006). This does not require highly relativistic speeds or extremely powerful jets, but it does require that in situ particle acceleration reaches electron energies of at least $\sim 30 \mathrm{TeV}$.

We are faced, therefore with the choice of fast and powerful jets or slow and multi-TeV particle accelerator jets. Recently, it was shown through Fermi observations that in the case of the archetypical quasar 3C 273 the inverse Compton model is ruled out (Meyer \& Georganopoulos 2014), favoring synchrotron from a second electron population as the X-ray emission mechanism (see also Cara et al. 2013, for the case of PKS 1136-135 studied through optical/UV polarimetry). Multi-wavelength observations of $3 \mathrm{C} 273$ hint at the two electron populations not being co-spatial (Jester et al. 2005). Understanding if this is indeed the case is imperative for understanding the physical conditions in the knots of these powerful jets, and high-dynamic-range deep radio-images from SKA1-MID (Bands 1-5) at resolutions up to 0.1 arcseconds (and SKA-LOW) are essential for mapping the spectral energy distribution of the extended jet as it extends out into the IGM.

\section{Feedback Between AGN Jets and their External Medium}

In the last few decades, observations of clusters of galaxies in the X-ray range revealed a lack of cool gas in the centres of many galaxies, contrary to expectations (see e.g. Fabian 2012, and references therein). This fact has not only important implications regarding the star formation rates in the galaxy and the growth of the central black hole (e.g. Cattaneo et al. 2009), but also regarding cold dark matter (CDM) models (Benson et al. 2003). The most accepted heating mechanisms proposed to stop the cooling flows are related to galactic activity or shocks produced during the merging of clusters. Among the former, the observed anti-correlation between the radio lobes formed by jets and the X-ray emission from the cluster gas suggests that the buoyancy of the under-dense cavities formed by the lobes could generate displacement work on the ambient gas, and could be responsible for the heating. Moreover, significant levels of metallicity have been recently detected at considerable distances from the active galaxy, which is possible only if an outflow has dragged those metals, produced from stars in the galaxy (e.g. Werner et al. 2011).

Recent observations have shown that the lobes are surrounded by shocks with low Mach numbers $(M \simeq 1-2)$ in powerful sources, i.e., they have not reached the buoyancy stage. This would 
imply faster evolution (pressure driven) and, accordingly, larger jet powers than estimated. Shocks may be extremely important and efficient in the heating process of the galactic and cluster gas because they can displace large amounts of gas from the host galaxy, quenching or triggering star formation (e.g. Perucho et al. 2014, and references therein). The discrepancy between CDM models, which predict a power law distribution of dark matter halos around galaxies, and the luminosity function of bright galaxies, which declines exponentially, could be also related to the feedback process (Benson et al. 2003). Recent semi-analytical work taking into account AGN feedback, reproduces the bright end of the luminosity function (e.g. Somerville et al. 2008).

It is clear that the dynamical impact of AGN jets within their host galaxies and environment can be very important to their evolution. The way in which the galactic activity relates to heating and cooling flows, or affects the evolution of the host galaxy, by changing the star formation rates and the growth of the central black hole, represents a fundamental problem in Astrophysics and Cosmology. Old populations of particles in the radio lobes formed by jets will certainly provide deep insight into the interaction between radio-jets and their environments. These particles emit at low radio frequencies and make SKA1-MID and SKA1-SUR (Bands $1-3$ ), as well as SKA1LOW, ideal instruments to detect them, and study how AGN jet activity impacts the evolution of the host galaxy and intra-cluster medium via the injection of particles and energy through relativistic jets (?). Angular resolutions on the order of the arcsecond ( $\sim 10$ arcseconds for SKA1-LOW), and sensitivities of the order of the $\mu \mathrm{Jy} / \mathrm{beam}$ in wide band continuum mode will be needed for these studies. The polarimetric capabilities of SKA will be relevant to study the influence of magnetic fields on the interplay of large scale-jets with their environment.

\section{AGN Jets Along Cosmic Time}

\subsection{The First AGN and their Supermassive Black Holes}

While AGN are common in the present-day Universe and SMBH with masses larger than $10^{9} \mathrm{M}_{\odot}$ were still reported at $z>6$ (e.g. Fan et al. 2006), it is natural to ask when the first AGN and their supermassive black holes started to form. In a seminal discussion by Rees (1978), a number of potential pathways, including single stars, stellar clusters and the direct collapse of very massive gas clouds have been considered. While the first stars are expected to have typical masses ranging from a few $10 \mathrm{M}_{\odot}$ to a few $100 \mathrm{M}_{\odot}$, the formation of stellar mass black holes from primordial stars seems only possible between $30-120 \mathrm{M}_{\odot}$ and $180-1000 \mathrm{M}_{\odot}$ (Heger et al. 2001). At least under the assumption of Eddington accretion, it therefore seems difficult that such stars could reach $10^{9} \mathrm{M}_{\odot}$ by $z \sim 6-7$ (e.g. Shapiro 2005).

More massive black hole seeds could be formed from the collapse of dense stellar clusters. Such clusters could form via dust-induced fragmentation in low-metallicity gas, with their collapse leading to characteristic black hole masses of a few times $1000 \mathrm{M}_{\odot}$ (Devecchi et al. 2012). The first simulations exploring the formation of massive black holes from the direct collapse of massive gas clouds have shown that strong radiation backgrounds are required to prevent the fragmentation into low-mass objects (Bromm \& Loeb 2003). Theoretical suggestions indicated that masses of $10^{4}-10^{5} \mathrm{M}_{\odot}$ could be reached from the low-angular momentum material in the first massive halos at $z \sim 10-15$. Numerical simulations indeed indicate the formation of massive self-gravitating 
disks in their centers, with high accretion rates of $\sim 1 \mathrm{M}_{\odot} \mathrm{yr}^{-1}$. The high accretion rates imply the formation of very massive protostars with cool atmospheres and weak radiative feedback, leading to a typical mass scale of $10^{5} \mathrm{M}_{\odot}$ (Schleicher et al. 2013).

These massive black holes are expected to lead to the formation of the first high-redshift quasars, which may contribute to the epoch of reionization. The pathways to observationally probe this population with SKA were outlined by Falcke et al. (2004). The compact radio cores of quasars with black hole masses of $10^{7} \mathrm{M}_{\odot}$ should be visible even at $z>10$. Due to the dense environments in the first galaxies, Falcke et al. (2004) predict that the black hole jet can potentially not break out from the galaxy, therefore leading to the formation of GHz-Peaked-Spectrum (GPS) sources (see also Taylor et al. 2015; Afonso et al. 2015). Early-Universe GPS sources are expected to be detected at low frequencies $(100-600 \mathrm{MHz})$ at sensitivities of $100 \mu \mathrm{Jy}$ and better. This makes wide bandwidth surveys on SKA1-LOW, and SKA1-MID/-SUR at Band 1, the best suited options for the detection of these sources. Also, HI spectroscopy via the $21 \mathrm{~cm}$ line, potentially allows for the detection of hundreds to thousands of bright QSOs via their HII regions at $z \sim 8-10$; see Wyithe et al. (2015) for an extended discussion. Later, SKA1-VLBI involving SKA-MID and SKA-SUR at the lowest practical frequencies (i.e. L Band, to maximize UV coverage with additional existing VLBI stations) are probably the right selection to confirm the compact nature of the GPS sources down to scales smaller than a few tens of milliarcsecond.

\subsection{Cosmological Studies of Radio-loud AGN. From $z \sim 0-10$}

Radio observations are the best-suited means to probe the evolution of radio jets in SMBH over cosmic time, as obscuration has a very low effect and largely unbiased surveys can thus be pursued (Falcke et al. 2004). The latter allows cosmological studies of black hole growth over a large range of black hole masses and cosmological redshifts. The NRAO VLA Sky Survey (NVSS, Condon et al. 1998) has detected 1.8 million sources in total intensity, with $14 \%$ of them showing a clear polarization signal of at least $3 \sigma$ (the majority of these being radio-loud AGN). While typical source counts have been restricted to flux density sensitivities of $\sim 10 \mu \mathrm{Jy}$ in total intensity (e.g. Windhorst 2003), bright sources above 100 mJy typically correspond to the radio-loud FRII sources, while a gradual transition towards FRI sources occurs around $30 \mathrm{mJy}$. At $\sim 1 \mathrm{mJy}$, an increasing fraction of radio sources is dominated by star formation rather than active galactic nucleus (see § 5.1), even though a contribution from a radio-quiet AGN may still be present.

An analysis of the polarization data of NVSS shows that the fractional polarization increases with decreasing flux density (Mesa 2002). This trend has been confirmed in subsequent studies and surveys (see Banfield et al. 2011, and references therein), including data from the Dominion Radio Astrophysical Observatory Deep Field polarization study and radio polarimetry of the ELAIS N1 Field. This intriguing anti-correlation clearly suggests a change in the magnetic field structure of the observed sources, even though the physical origin of this behaviour is not yet understood. To investigate the potential origin of the polarization, Shi et al. (2010) studied a sample of highly polarized objects. In their sample, sources with more than $30 \%$ polarization were contained in elliptical galaxies with $1.4 \mathrm{GHz}$ luminosities in the range of $10^{23}-10^{24} \mathrm{~W} \mathrm{~Hz}^{-1}$. As they reported no dependence on optical morphology, redshift, linear size and radio power, the high polarization seems likely a result of intrinsic properties of the source (see also Stil et al. 2014). 
The deep total flux and polarimetric surveys that are being suggested by the SKA Magnetism and Continuum Science Working Groups for AGN at SKA1-MID and SKA1-SUR, mainly on Bands 2 and 3 (Taylor et al. 2015; Johnston-Hollitt et al. 2015; Prandoni et al. 2015; Smolcic et al. 2015), will allow further investigations regarding the total and polarized synchrotron emission of AGN. While a measurement of the spectra help to disentangle the contributions of AGN and star formation and provide information about the AGN environment, the measurement of the polarized fractions allows to further investigate the anti-correlation between radio flux and polarization fraction, and to potentially relate it to the power of the central engine, reflected in the jet. Whereas previous studies have found no explicit redshift dependence in the observed correlation up to $z \sim 3$, the far deeper SKA1 surveys are expected to probe radio fluxes to $z \sim 10$ where correlations with redshift cannot be ruled out.

On a second stage, deep follow-up SKA1-VLBI surveys of AGN will have the capability to image much smaller scales to investigate the origin of the I-p anti-correlation in terms of cancellation of orthogonal polarized regions on bright sources. These VLBI surveys will also allow us for studies of the brighter spatially resolved jets on cosmological scales, which will certainly help to provide information on the environments (through VLBI morphological studies). At L band, a 5 min-long VLBI observation including SKA1-MID will achieve a $5 \sigma$ level of $25 \mu \mathrm{Jy}$. This can allow to observe $\sim 1000$ sources in less than 100 hours of observation. The value of these VLBI surveys will be much enhanced by synergies with deep optical surveys such as SDSS (York et al. 2000), and LSST (Bacon et al. 2015).

\section{Identifying AGN Populations from SKA1 Surveys}

Two main processes contribute to the extragalactic continuum radio emission at intermediate radio frequencies (i.e. 1.4 to $5 \mathrm{GHz}$ ): the non-thermal emission associated with relativistic electrons powered by AGN and a combination of free-free and synchrotron emission from the star formation activity in galaxies. The bright radio sky is dominated by the emission driven by radio-loud AGN where the presence of compact or slightly resolved radio cores on milliarcsecond-scale are the best indicators of the AGN activity. At faint flux densities $(<0.5-1 \mathrm{mJy})$ the contribution from star-forming galaxies to the radio source population becomes increasingly important (e.g. Seymour et al. 2008; Padovani et al. 2011, and Fig. 2). Moreover, recent work has confirmed that at 10-100 micro-Jy levels, the radio-quiet AGN are not radio-silent (e.g. Bonzini et al. 2012).

Linear polarization and spectral index information from the massive surveys on SKA1-MID and SKA1-SUR described above will be a useful tool to disentangle AGN jet emission from that of star-forming galaxies, thus providing a first selection of AGN candidates from large SKA commensal surveys. Clearly, the optical data and photometric redshifts measured by the Large Synoptic Survey Telescope (LSST) will be an invaluable complement for any kind of SKA survey involving extragalactic sources, e.g. Bacon et al. (2015), and specifically for all those studies interested in radio emitting objects at all ranges of redshift. In particular, the optical photometric data from LSST will also be extremely useful in discriminating between the AGN and star forming galaxy populations. A definitive probe of the presence of AGN radio loud activity will be the detection of high brightness temperature from embedded radio-AGN cores in the host galaxies through 
ultra-high-resolution (milliarcsecond or sub-milliarcsecond scale) SKA1-VLBI wide-field-of-view observations, see Paragi et al. (2015).

Adding to the present High Sensitive Array (HSA), the SKA1-MID and/or SKA1-SUR as a phased array, will obtain an angular resolution better than 5 milliarcseconds at $\mathrm{L}$ Band, which corresponds to $10-40 \mathrm{pc}$ in the redshift range 0.1 to 5 . This high angular resolution will be coupled with the exceptional sensitivity provided by the SKA1. One hour of observation with the HSA + SKA1-MID at L Band at a recording rate of $4096 \mathrm{Mb} / \mathrm{s}$ will allow to reach $0.75 \mu \mathrm{Jy} / \mathrm{beam}$ $(0.84 \mu \mathrm{Jy} /$ beam without Arecibo). For less demanding sensitivities, similar VLBI observation will achieve a sensitivity of $\sim 5 \mu \mathrm{Jy} /$ beam in $5 \mathrm{~min}$ integrations. This high sensitivity - high angular resolution will allow not only identifying starburst galaxies, and resolving and mapping the region of star-formation, but also imaging jets at parsec scales in the inner region of AGN up to high $z$.

\section{Studies of Relativistic Jets in AGN with SKA1: A Summary}

Previous sections outline a variety of relevant science cases for cutting-edge studies of relativistic jets in AGN that can be performed with SKA1. In particular, the superb sensitivity of SKA1 will allow us to detect massive samples of AGN from commensal full-polarization all-sky and deep surveys performed at both SKA1-MID and SKA1-SUR at frequencies between $\sim 1$ and $3 \mathrm{GHz}$ (Taylor et al. 2015; Johnston-Hollitt et al. 2015; Prandoni et al. 2015; Smolcic et al. 2015). Identification of AGN in these surveys may be performed in different and complementary ways by using classification arguments based on linear polarization, spectral index, optical photometry, and brightness temperature measurements. Linear polarization and spectral index information will be available from SKA commensal surveys. Optical photometric and redshift data from the LSST will be of invaluable help, whereas the highly-constraining brightness-temperature information will be provided by follow up wide-field VLBI observations involving SKA1, ideally including both the phased SKA1-MID and SKA1-SUR at L-band, where UV coverage can be optimized by including a large number of non-SKA VLBI stations. The wide field requirement needs multiple $(\geq 4)$ beam formers for the SKA1-MID and SKA1-SUR cores, see Paragi et al. (2015). These cores will dominate the sensitivity of the entire VLBI array, and can therefore reliably detect high brightness temperatures from AGN at a few $10 \mu \mathrm{Jy}$ level or better.

The data from the above-mentioned full-polarimetric surveys at SKA1-MID and SKA1-SUR will be of almost inestimable value for essentially all AGN science cases described in this chapter, specially for radio-loud/radio-weak, and massive cosmological statistical-studies. Investigations of the feedback between large scale AGN jets and their intergalactic medium, and those for the search of the first GPS-like radio AGN and black holes, will also benefit from these enormous survey datasets, as well as from lower frequency all-sky programs at SKA-LOW.

The non-isotropic character of radio AGN, together with the fact that some of the most pressing open questions about AGN are related to their innermost (pc and sub-pc) jet regions, makes ultra-high angular resolution VLBI observations an essential ingredient of next-generation studies. In particular, jet formation, collimation, and acceleration studies will only be complete with the aid of additional super-sensitive full-polarization VLBI observations at multiple observing frequencies up to $\sim 15 \mathrm{GHz}$, which will only be possible with SKA1-MID on Band 5 and lower. These observations are also mandatory for detailed studies of the velocity field and magnetic field properties of 
the innermost AGN jet regions, as well as for research on the high energy emission site and emission mechanism in combination with other multi-spectral range facilities operating simultaneously in the southern hemisphere.

Characterizing the jet composition and magnetic field, and measuring the low-energy cut-off of the relativistic electron energy distribution through circular polarization analysis requires not only multi-frequency (up to Band 5) SKA1-MID, and SKA1-VLBI observations to map the different regions of the AGN, but also -and even more importantly- requires high precision circular and linear polarization measurements up to $\sim 0.01 \%$, and $\sim 0.1 \%$, respectively, that can be achieved with linear feeds as planned for SKA1-MID. Great attention must be paid at design time to ensure optimum performance in all four Stokes parameters so that the scientific potential of SKA can be realized in these areas.

\section{Considerations for SKA1 Early Science and SKA2}

A reduction by $\sim 50 \%$ in sensitivity during the early-science stage of SKA1 will not dramatically affect most of the science use cases presented here as long as SKA1 receivers retain adequate polarization purity as described above. In particular, the proposed research on jet formation, collimation and acceleration; jet composition, precise characterization of jet parameters and jet emission properties; jet feedback; and the identification of AGN populations from early-science SKA surveys will provide excellent science outcomes even with a large reduction in sensitivity while waiting for the complete SKA1 to be finished.

The requirements for detailed imaging studies of FRII radio galaxies outlined in $\S$ 6, i.e. $\sim 0.01$ arcsec resolution, $\sim 10 \mathrm{nJy} /$ beam, and $>10^{7}: 1$ dynamic range at bands 4 and 5 of SKA1-MID, will only be achieved by SKA2. Certainly, the ability of SKA-MID to achieve frequencies up to $24 \mathrm{GHz}$ at the stage of the SKA2 will greatly boost ultra-high-resolution VLBI studies to probe the innermost jet regions of nearby sources, where the jet is still being collimated and accelerated.

During the final stage of SKA, the possibility of SKA2-MID observations including a number of very large ( $\gtrsim 1000 \mathrm{~km}$ ) baselines employing a homogeneous array of SKA stations would open a new era of extremely high-dynamic-range VLBI observations with the SKA alone (Garrett 1999). These high dynamic-range observations would allow imaging of an unprecedented number of jetcounterjet systems, and their transverse structure, which will certainly allow for extremely high precision estimates of the actual physical conditions at the innermost regions of jets, close to their formation and collimation sites. Of paramount importance for these SKA2-VLBI observations is the availability of quasi-scaled arrays over as wide a range of frequencies as possible to avoid changing resolution with frequency, which is crucial to eliminate resolution biases on the relevant polarization studies described in $\S 2,3,4$, and 6 .

\section{References}

Afonso, J., et al. 2015, "Identifying the first generation of radio powerful AGN in the Universe with the SKA" in proc. Advancing Astrophysics with the SKA, PoS(AASKA14)071

Agudo, I., et al. 2011, ApJ, 726, L13

Aloy, M. A., et al. 2000, ApJ, 528, L88

Asada K., et al. 2008, ApJ, 675, 79 
Asada K., et al. 2014, ApJ, 781, L2

Bacon, D., et al. 2015, "Synergy between the Large Synoptic Survey Telescope and the Square Kilometre Array" in proc. Advancing Astrophysics with the SKA, PoS(AASKA14)145

Banfield, J. K. et al. 2011, ApJ, 733, 69

Benson, A.J., et al., 2003, ApJ, 599, 38

Blandford, R. D. \& Znajek, R. L., 1977, MNRAS, 179, 433

Blandford, R. D. \& Payne, D. G., 1982, MNRAS, 199, 883

Bonzini et al. 2012, ApJS 203, 15

Bonzini et al. 2013, MNRAS 203, 15

Broderick, A. E., \& McKinney, J. C., 2010, ApJ, 725, 750

Bromm, V. \& Loeb, A. 2003, ApJ, 596, 34

Cara, M., et al. 2013, ApJ, 773, 186

Carilli, C. L., \& Rawlings, S. 2004, New Astronomy Reviews, 48, 979

Cattaneo, A., et al., 2009, Nature, 460, 213

Celotti, A., Ghisellini, G., \& Chiaberge, M. 2001, MNRAS, 321, L1

Chartas, G., et al. 2000, ApJ, 542, 655

Condon, J. J., et al. 1998, AJ, 115, 1693

Devecchi et al. 2012, MNRAS, 421, 1465

York, D. G. et al. 2000, AJ, 120, 1579

Fabian, A.C., 2012, ARA\&A, 50, 455

Falcke, H., Körding, E. \& Nagar, N. M. 2004, New Astronomy Reviews, 48, 1157

Fan et al. 2006, AJ, 131, 1203

Fanaroff, B. L., \& Riley, J. M. 1974, MNRAS, 167, 31P

Fender, R., et al. 2015, "The Transient Universe with the Square Kilometre Array", in proc. Advancing Astrophysics with the SKA, PoS(AASKA14)051

Finke, J. D., Razzaque, S., \& Dermer, C. D., 2010, ApJ, 712, 238

Gallimore, J. F., et al. 2006, AJ, 132, 546

Garrett, M. A. 1999, in proc. "Perspectives on Radio Astronomy: Science with Large Antenna Arrays", 139

Ghisellini G., et al., 2010, MNRAS, 405, 387

Ghisellini G., et al. 2014, MNRAS, 438, 2694

Giroletti, M., et al. 2015, "The connection between radio and high energy emission in black hole powered systems in the SKA era", in proc. Advancing Astrophysics with the SKA, PoS(AASKA14)153

Gómez, J. L., et al. 2011, ApJ, 733, 11

Gómez, J. L., et al. 2012, International Journal of Modern Physics, 8, 265

Gopal-Krishna, \& Wiita, P. J. 2000, A\&A, 363, 507

Harris, D. E., \& Krawczynski, H. 2006, ARAA, 44, 463

Heger et al. 2001, NuPhA, 688, 197

Homan, D., et al. 2009, ApJ, 696, 328

Jester, S., et al. 2005, A\&A, 431, 477

Johnston-Hollitt, M., et al. 2015, "Using SKA Rotation Measures to Reveal the Mysteries of the Magnetised Universe", in proc. Advancing Astrophysics with the SKA, PoS(AASKA14)092

Jorstad, S. G., et al., 2005, AJ, 130, 1418

Komissarov, S. S., et al. 2007, MNRAS, 380, 51

Kovalev Y.Y., et al. 2007, ApJ, 668, L27

Laing R.A., 1981, ApJ, 248, 87

Laing, R. A. \& Bridle, A. H., 2002, MNRAS, 336, 1161

Laing, R. A. 2015, "Kinematics and Dynamics of kiloparsec-scale Jets in Radio Galaxies with SKA", in proc. Advancing Astrophysics with the SKA, PoS(AASKA14)107 
Laing, R. A. \& Bridle, A. H., 2014, MNRAS, 437, 3405

Li, Z.-Y., Chiueh, T. \& Begelman, M. C., 1992, ApJ, 394, 459

Lister, M. L., et al., 2009, AJ, 138, 1874

Lister M.L., et al., 2013, AJ, 146, 120

Lobanov, A. P. \& Zensus, J. A. 2001, Science, 294, 5540

Marscher, A. P., et al. 2010, ApJ, 710, L126

McNamara, B. R., Rohanizadegan, M., \& Nulsen, P. E. J. 2011, ApJ, 727, 39

McKinney, J.C., Tchekhovskoy, A. and Blandford, R.D., 2012, MNRAS, 423, 3083

Mesa, D. 2002, A\&A, 396, 463

Meyer, E. T., \& Georganopoulos, M. 2014, ApJ, 780, L27

O'Sullivan S., et al. 2013, MNRAS, 435,311

Padovani, P., et al. 2011, ApJ, 740, 20

Paragi, Z., et al. 2015, "Very Long Baseline Interferometry with the SKA", in proc. Advancing Astrophysics with the SKA, PoS(AASKA14)143

Perucho, M., et al., 2014, submitted.

Prandoni, E., et al. 2015, "Revealing the Physics and Evolution of Galaxies and Galaxy Clusters with SKA Continuum Surveys", in proc. Advancing Astrophysics with the SKA, PoS(AASKA14)067

Rawlings S. \& Saunders R., 1991, Nature, 349, 138

Rees, M. J. 1978, Phys. Scr., 17, 193

Sbarrato T., et al. 2012a, MNRAS, 421, 1764

Sbarrato T., et al. 2012b, MNRAS, 426, L91

Schleicher, D.R.G. et al. 2013, A\&A, 558, 59

Seymour et al. 2008, MNRAS 386, 1695

Shapiro, S. 2005, ApJ, 620, 59

Shi, H. et al. 2010, MNRAS, 409., 821

Sikora, M., Begelman, M. C., \& Rees, M. J. 1994, ApJ, 421, 153

Sironi, L., \& Spitkovsky, A. 2014, ApJL, 783, L21

Smolcic, V., et al. 2015, "Exploring AGN Activity over Cosmic Time with the SKA", in proc. Advancing Astrophysics with the SKA, PoS(AASKA14)069

Somerville, R.S., et al., 2008, MNRAS, 391, 481

Sopp, H.M., \& Alexander, P. 1991, MNRAS, 251, 14P

Stawarz, Ł., \& Petrosian, V. 2008, ApJ, 681, 1725

Stil, J. M. et al. 2014, ApJ, 787, 99

Summerlin, E. J., \& Baring, M. G., 2012, ApJ, 745, 63

Schwartz, D. A., et al. 2000, ApJ, 540, L69

Tavecchio, F., et al. 2000, ApJ, 544, L23

Taylor, R., et al. 2015, "SKA Deep Polarization and Cosmic Magnetism", in proc. Advancing Astrophysics with the SKA, PoS(AASKA14)113

Tchekhovskoy, A., Narayan, R., \& McKinney, J.C. 2011, MNRAS, 418, L79

Ulvestad, J.S., Antonucci, R.R.J., \& Barvainis, R. 2005, ApJ, 621, 123

Vlahakis, N. \& Königl, A., 2004, ApJ, 605, 656

Volonteri M., et al., 2011, MNRAS, 416, 216

Werner, N., et al., 2011, MNRAS, 415, 3369

Windhorst, R. A. 2003, New Astronomy Reviews, 47, 357

Wyithe, S., et al. 2015, "Imaging HII Regions from Galaxies and Quasars During Reionisation with SKA", in proc. Advancing Astrophysics with the SKA, PoS(AASKA14)015 